Enhancement of nol ecul ar i nt er act i ons i $n$ strongl y scattering di el ectric composite opt i cal medi a

\begin{tabular}{|l|l|}
\hline 著者 & Tomit a Nakot o, Ohosum Kazumasa, I kar i H roshi \\
\hline $\begin{array}{l}\text { j our nal or } \\
\text { publ i cat i on ti tl e }\end{array}$ & Physi cal Revi ew B \\
\hline vol une & 50 \\
\hline number & 14 \\
\hline page r ange & $10369-10372$ \\
\hline year & 1994 10 \\
\hline 出版者 & Aner i can Physi cal Soci et y \\
\hline 権利 & (c) 1994 The Aner i can Physi cal Soci et y \\
\hline URL & ht t p: //hdl . handl e. net /10297/575 \\
\hline
\end{tabular}




\title{
Enhancement of molecular interactions in strongly scattering dielectric composite optical media
}

\author{
Makoto Tomita and Kazumasa Ohosumi \\ Department of Physics, Faculty of Science, Shizuoka University 836, Ohya, Shizuoka City 422, Japan \\ Hiroshi Ikari \\ Department of Physics, Faculty of Education, Shizuoka University 836, Ohya, Shizuoka City 422, Japan
}

(Received 17 May 1994)

\begin{abstract}
We observed fast nonexponential luminescence decay from a molecular dye in a suspension of dyed polystyrene spheres when both the interparticle distance and the sphere size is comparable to the wavelength of the emission. This nonexponential lifetime depends on the square root of the time, suggesting a resonance dipoledipole interaction associated with the Mie resonances. This phenomenon is interpreted in terms of resonance energy transfer between molecules leading to the formation of a photonic band mode.
\end{abstract}

Quantum electrodynamics of atoms and molecules interacting with photons in a class of strongly scattering dielectric materials has been extensively studied. ${ }^{1-5}$ These dielectrics consist of an ordered or nearly ordered array of spherical scatterers with high refractive index and exhibit a photonic band gap or pseudogap. ${ }^{6-8}$ For an isolated atom with a transition frequency in the photonic band gap, spontaneous emission is inhibited ${ }^{1,6}$ and a quantum-electrodynamic photon state bound to the atom is formed. ${ }^{3}$ When a collection of identical impurity atoms is placed within the photonic band gap, the localized photon can hop to the unexcited atom by the resonance dipole-dipole interaction (RDDI). This leads to the formation of a narrow photonic impurity band. This impurity band exhibits different kinds of nonlinear optical properties. ${ }^{3}$ Along with these studies of quantum electrodynamics, there has also been interest in photoluminescence or chemical interactions modified by electromagnetic modes. ${ }^{9}$

Experimental observations of a change in the luminescence lifetime of dye molecules in dielectric media were reported by Martorell and Lawandy. ${ }^{1}$ They measured the spontaneous emission lifetime from a molecular dye in crystallized and colloidal suspensions of monodispersed polystyrene spheres. In their experiments it has been argued that the dye molecules stay in water, away from the polystyrene particles. Recent experimental results for similar dielectric media by Tong et al., however, suggest otherwise. They reported that most dye molecules are either in, on, or in the near neighborhood of the monodispersed polystyrene particles, and this chemical-surface interaction causes the observed increase in the luminescence lifetime of dye molecules in a colloidal suspension of polystyrene spheres. ${ }^{10}$ With such chemical-interaction effects excluded, the change in the luminescence lifetime of the dye in the colloidal suspensions may be a result of the quantum electrodynamics effect, which has attracted much attention in recent years.

Here we present a preliminary analysis of luminescence lifetime measurements in colloidal suspensions of dyed polystyrene spheres. In contrast to the previous measurements of the luminescence lifetime, the dyed polystyrene spheres, in our measurements, were used where the dye molecules are incorporated in the polystyrene spheres. Consequently, the chemical-interaction effect may be excluded and there re- main observed changes that bear a plausible relation to the quantum electrodynamics of photon-field effect on the radiation source.

We used six kinds of suspensions of rhodamine-doped polystyrene spheres in water. The diameters are $D=0.0481$ $\mu \mathrm{m}, 0.11 \mu \mathrm{m}, 0.21 \mu \mathrm{m}, 0.489 \mu \mathrm{m}, 0.993 \mu \mathrm{m}$, and 1.89 $\mu \mathrm{m}$. The standard deviations vary from 0.8 to $4.7 \%$ depending on the sphere size. The rhodamine was incorporated when styrene monomers were polymerlized (Polyscience Inc.). With some part of dye molecules being left undoped, the best estimation of the dye concentration in the polystyrene is $0.75 \pm 0.25 \mathrm{wt}$. \%, which corresponds to the intermolecular distance from 27 to $34 \AA$. The excitation light source was the second harmonics of a $\mathrm{Nd}^{3+}$ YAG (yttrium aluminum garnet) laser with a wavelength of $532 \mathrm{~nm}$ and a repetition rate of $82 \mathrm{MHz}$. The laser beam of $10 \mathrm{~mW}$ in average power was focused on the sample cell of $1 \mathrm{~mm}$ in thickness. The luminescence from the sample cell window on which the incident laser beam was focused was led to a monochrometer and detected by a microchannel plate photomultiplier tube. The detected signal was analyzed using a time-correlated single-photon-counting system. In some experiments, the luminescence was also examined in the transmission-type geometry, and the same results were obtained. The temporal resolution of the system was 65 psec.

Figure 1 shows the time dependence of the luminescence lifetime for spheres of $0.993 \mu \mathrm{m}$ in diameter for different wavelengths. The intersphere distance is $d=850 \mathrm{~nm}$, where $2 d$ is the average distance between the centers of neighboring spheres. In addition to the intrinsic slow decay in the isolated dye molecule, the anomalous fast nonexponential decay appears. We tried to find the origin of this fast decay. Measurements of the excitation power dependence of the luminescence have been performed. The resultant luminescence intensity was proportional to the excitation power. When the excitation power was also increased by a factor of 1000 , the time dependence of the luminescence was unchanged. Therefore, it appears that the fast decay component is not a laser oscillation ${ }^{11}$ or a stimulated emission. ${ }^{2}$ This fast decay was observed only in spheres larger than $0.21 \mu \mathrm{m}$ in diameter, whereas it was not observed in spheres of 0.048 and $0.11 \mu \mathrm{m}$ in diameter. For spheres of 0.048 and 0.11 


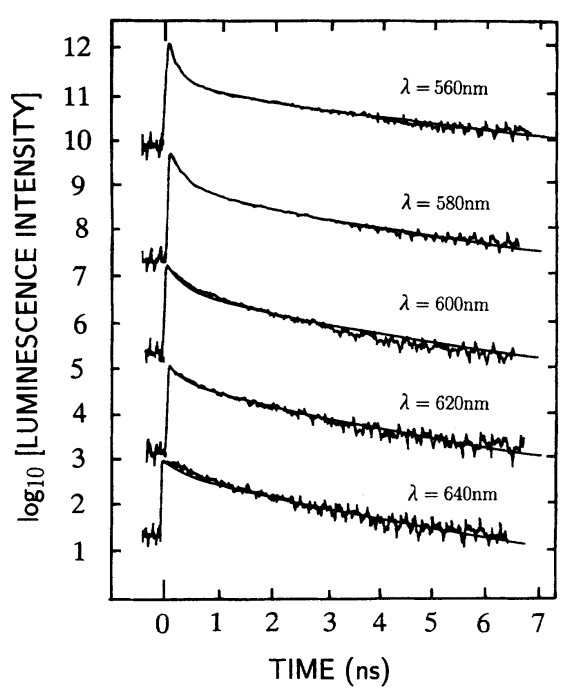

FIG. 1. The time-resolved luminescence for spheres of 0.993 $\mu \mathrm{m}$ in diameter for different wavelengths.

$\mu \mathrm{m}$ the luminescence decay curve was well described by a single exponential function with the lifetime of $3.2 \mathrm{nsec}$. In our experiments of dyed polystyrene sphere, the slow lifetime in spheres of 0.048 and $0.11 \mu \mathrm{m}$ was unchanged with the intersphere distance varying from 40 to $800 \mathrm{~nm}$, which is in contrast to a previous report ${ }^{5}$ where dye molecules were dissolved in the water. At the wavelength observed, the diameters 0.048 and $0.11 \mu \mathrm{m}$ are smaller than the minimum size required for the Mie resonances (Rayleigh limit). Therefore, it shows that the observed fast decay is associated with the Mie resonance of spheres.

The importance of the cooperative effect of neighboring spheres is clearly demonstrated in Fig. 2, where the dependence of the time-resolved luminescence on the intersphere distance is shown for the spheres of $0.993 \mu \mathrm{m}$ in diameter. The relative intensity of the fast decay component decreases with the intersphere distance increase. Figure 3 shows the luminescence spectra for the sphere of $0.993 \mu \mathrm{m}$ in diameter.

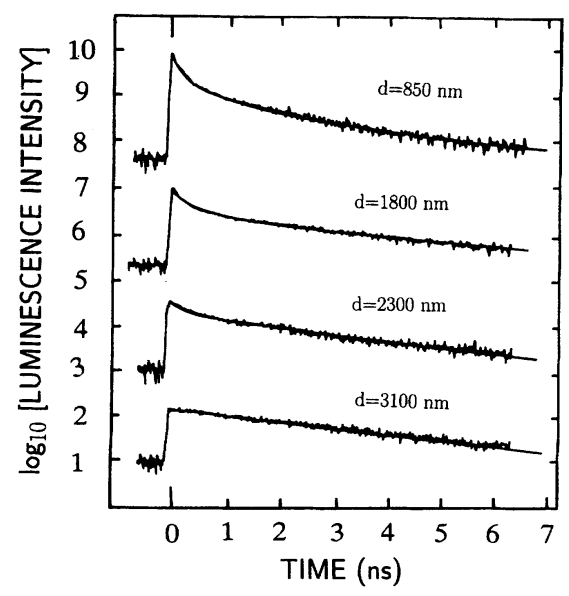

FIG. 2. The time-resolved luminescence for spheres of 0.993 $\mu \mathrm{m}$ for different intersphere distances.

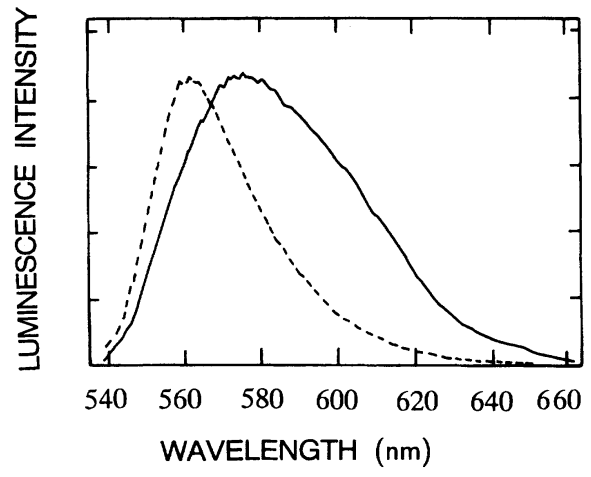

FIG. 3. Luminescence spectra for $0.993 \mu \mathrm{m}$ spheres. The solid line is for the intersphere distance $d=850 \mathrm{~nm}$ and the dotted line is for $d=180 \mathrm{~nm}$.

The wavelength in the peak of the luminescence spectra is shifted to red at the short intersphere distance where the fast decay component appears. This shift is qualitatively related to the relative intensity of the fast decay component. We also investigated aggregated dyed polystyrene spheres of 0.21 , $0.489,0.993$, and $1.89 \mu \mathrm{m}$, which were prepared by drying the water from the original samples. The resultant relative refractive index between polystyrene and the surrounding air becomes large enough to be 1.60. The fast decay component, however, was not observed in all these samples. From this result, it is considered that our fast decay does not originate from the contact or aggregation of spheres. It may be suggested that the high $Q$ factors of resonant modes of individual spheres are reduced by the contact or aggregation of nearby spheres.

We consider the resonance energy as the origin of the fast decay component and explain the experimental data by the following equation (1):

$$
I(t)=\exp \left[-\left(t / \tau_{0}\right)\right]\left\{(1-\alpha)+\alpha \exp \left[-\gamma\left(t / \tau_{0}\right)^{1 / 2}\right]\right\},
$$

where $\tau_{0}$ is the intrinsic lifetime of rhodamine in bulk polystyrene and is 3.2 nsec. Equation (1) represents the energy transfer by dipole-dipole interaction when acceptor molecules are randomly distributed in space. ${ }^{12,13}$ In a free space with dielectric constant $\epsilon$, the factor $\gamma$ is represented as

$$
\gamma=\Gamma(1 / 2) C\left\{\frac{4 \pi \tau_{0}\left|M_{e g}\right|^{2}\left|M_{g e}\right|^{2}}{27 \hbar \epsilon^{2}} \int f_{a}(\nu) f_{b}(\nu) d \nu\right\}^{1 / 2},
$$

where $\left|M_{e g}\right|$ and $\left|M_{g e}\right|$ are dipole moments, $C$ is the concentration of molecules, and $f_{a}(\nu)$ and $f_{b}(\nu)$ are the absorption and the emission spectra, respectively. ${ }^{13}$ The first term in Eq. (1) denotes the intrinsic decay process of the isolated dye molecule in free space, while the second term is attributed to the energy transfer between molecules in the strongly scattering media. In general, the transferred energy by RDDI is dissipated through nonradiative energy relaxation processes in the molecular systems. Here, we will not discuss the details of the energy dissipation processes after the resonance energy transfer. The critical transfer distance for rhodamine 6 G in bulk glycerol is reported to be $39 \AA .{ }^{14}$ This value is comparable to our intermolecular distance, suggesting our 


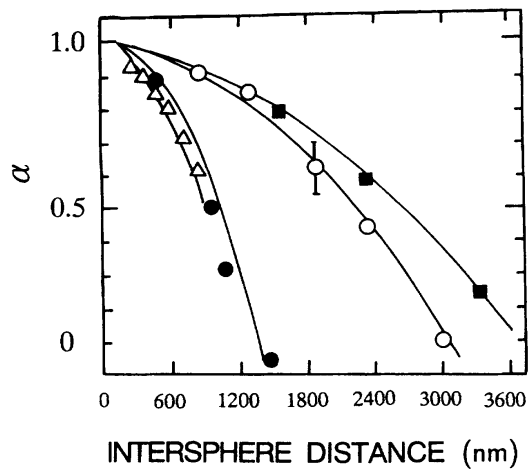

FIG. 4. The relative intensity of the fast decay component as a function of the intersphere distance, for spheres for $\Delta, 0.21 \mu \mathrm{m}$; ○, $0.489 \mu \mathrm{m}$; $\bigcirc, 0.993 \mu \mathrm{m}$; and $\mathbf{\square}, 1.89 \mu \mathrm{m}$.

fast decay originates from RDDI. The major fraction in our time dependence of the luminescence decay may be caused by the nearby molecules within a single sphere, since the dipole-dipole interaction is rapidly attenuated with the intermolecular distance increase. The resonance energy is, however, also expected to be transferred between dye molecules in the neighboring spheres. Inside the medium, there exists a dark and bright irregular pattern referred to as volume speckle, which is due to the random interference of the incident coherent light. This interference pattern suggests that the unexcited spheres exist in nearly the same number as excited spheres when the sphere size is comparable to the wavelength. Therefore, the energy transfer from excited spheres to unexcited spheres may effectively occur. In Fig. 1, the fitting curves calculated on the basis of Eq. (1) are plotted together with the experimental results. Parameters used are $\gamma=5.2,3.8,2.6,2.3$, and 2.2 for $\lambda=560,580,600,620$, and $640 \mathrm{~nm}$, respectively. The factor $\alpha=0.89$ is common to all wavelengths. The relative intensity of the fast decay component is independent of the wavelength, whereas the decay is faster in the short-wavelength regions. The spectral overlapping factor in Eq. (1) denotes the observed wavelength dependence of $\gamma$, which may be subject to the dispersion relation of an electromagnetic mode in the polystyrene suspensions. This spectral factor may also explain the change in the luminescence spectra in Fig. 3. The calculated curves for the intersphere distance of $\alpha$ are plotted in Fig. 2 together with the experimental results. The parameter $\gamma$ is 3.8 throughout all curves, while $\alpha=0.89,0.71,0.45$, and 0 for $d=850,1800,2300$, and $3100 \mathrm{~nm}$, respectively. The relative intensity of the fast decay component decreases with the intersphere distance increase, whereas the decay rate is not changed. The dependence of $\alpha$ on the intersphere distance is shown in Fig. 4 for different sphere diameters.

It is suggested that the observed fast decay originates from a cooperative effect in a large number of spheres. In this connection, it is of interest to understand our experimental results in terms of the tight-binding photonic band model, because the tight-binding approximation is a good approach for the band structure in some electric crystals, such as alkali metals, where each atom in the crystal still retains the main characteristics of an isolated state. Each sphere is dressed with evanescent light in the range of the order of the wavelength. Every neighboring sphere mode may couple when the evanescent modes are overlapped, resulting in a macroscopic photonic band mode throughout the medium. Impurity molecules would strongly couple with this photonic band mode and interact with the surrounding impurity-sphere systems. This mechanism will result in a cooperative response in the system. We introduce a parameter $\kappa$, which represents the extinction length of the evanescent light. We then assume that the macroscopic band mode is developed when the centers of two spheres approach within the interaction region of $D+2 \kappa \lambda$, where $\lambda$ is the wavelength. We consider a model in which the parameter $\alpha$ in Eq. (1) is represented by the probability that a particular sphere couples with at least one other sphere. The parameter $\kappa$ is estimated to be 1.3, 1.5, 3.1, and 2.6 for spheres of $0.21,0.48,0.993$, and $1.89 \mu \mathrm{m}$, respectively, from the intersphere distance $d_{1 / 2}$, where $d_{1,2}$ is the distance where $\alpha$ becomes 0.5 in Fig. 4 . We then find that the extinction length of the evanescent light becomes long with the sphere size increase. The enhancement of the molecular interaction in strongly scattering media is the manifestation of the breakdown of the independent scattering approximation. Therefore, we may compare the interaction distance $d_{1 / 2}$ with the influence diameter for which Mie resonances appear. Using the Lorentz Mie theory, ${ }^{15}$ the normalized scattering cross sections in the absence of absorption are calculated as $\sigma=0.11,0.93,2.9$, and 3.0 for spheres of 0.21 , $0.489,0.993$, and $1.89 \mu \mathrm{m}$ in diameter, respectively, which corresponds to the influence diameter $D^{\prime}$, where $\pi\left(D^{\prime} / 2\right)^{2}=\sigma \pi(D / 2)^{2}$, and $D^{\prime}=0.07,0.47,1.7$, and 3.3 $\mu \mathrm{m}$, respectively. These rough estimations are in qualitative agreement with the experimental results. Contributions from molecules coupled with the band mode are given by the parameter $\alpha$ in Eq. (1), whereas there exist also molecules coupled with the propagating mode. Therefore, at the fixed wavelength, the band mode and the modified free space mode coexist. The lower-energy part of the band mode may correspond to the energy of the fundamental morphology dependent resonance. It is seen that the RDDI is inhibited below this tight-binding photonic band edge in our system.

In conclusion the observed changes in the luminescence lifetime in a collection of dyed spheres are attributed to the enhancement of the molecular interaction in strongly scattering media. However, in order to study the effect related to quantum electrodynamics, it is important to isolate all contributions from the chemical interactions. Further measurements are in progress to resolve the many factors that can contribute to the observed changes in the luminescence lifetime and spectra of dye molecules in colloidal suspensions of dyed polystyrene spheres. It is suggested that resonant energy transfer occurring in the photonic band gap may result in new types of nonlinear optical phenomena such as second harmonics generation. ${ }^{3}$ Therefore, it may be interesting to investigate the energy dissipation processes following RDDI. In suspensions of polystyrene spheres, a local periodic structure exists in a macroscopic disordered structure. ${ }^{16}$ It is also of interest to investigate RDDI between impurity systems placed in periodic dielectric structures where the restriction in the phase space is more clear. 
${ }^{1}$ J. Martorell and N. M. Lawandy, Phys. Rev. Lett. 65, 1877 (1990).

${ }^{2}$ A. J. Campillo, J. D. Eversole, and H.-B. Lin, Phys. Rev. Lett. 67, 437 (1991).

${ }^{3}$ S. John and J. Wang, Phys. Rev. Lett. 64, 2418 (1990); Phys. Rev. B 43, 12772 (1991).

${ }^{4}$ G. Kurizki and A. Z. Genack, Phys. Rev. Lett. 61, 2269 (1988).

${ }^{5}$ J. Martorell and N. M. Lawandy, Phys. Rev. Lett. 66, 887 (1991).

${ }^{6}$ E. Yablonovitch, J. Opt. Soc. Am. B 10, 283 (1993).

${ }^{7}$ E. Yablonovitch, T. G. Gmitter, R. D. Mead, K. D. Brommer, A. M. Rappe, and J. D. Joannopoulos, Phys. Rev. Lett. 67, 3380 (1991).

${ }^{8}$ S. John, Phys. Rev. Lett. 53, 2169 (1984); M. P. van Albada and
A. Lagendijk, ibid. 55, 2696 (1985); P. E. Wolf and G. Maret, ibid. 55, 2692 (1985).

${ }^{9}$ P. Das and H. Metiu, J. Phys. Chem. 89, 4680 (1985).

${ }^{10}$ B. Y. Tong, P. K. John, Y. Zhu, Y. S. Liu, S. K. Wong, and W. R. Ware, J. Opt. Soc. Am. B 10, 356 (1993).

${ }^{11}$ M. K. Gonokami, K. Takeda, H. Yasuda, and K. Ema, Jpn. J. Appl. Phys. 31, L99 (1992).

${ }^{12}$ K. B. Eisenthal and S. Siegel, J. Chem. Phys. 41, 652 (1964).

${ }^{13}$ T. Förster, Discuss. Faraday Soc. 27, 7 (1959).

${ }^{14}$ K. B. Eisenthal, Chem. Phys. Lett. 6, 155 (1970).

${ }^{15}$ G. Mie, Ann. Phys. (Leipzig) 25, 377 (1908).

${ }^{16}$ N. Ise, T. Okubo, M. Sugimura, K. Ito, and H. J. Nolte, J. Chem. Phys. 78, 536 (1983). 